\title{
Analysis of benzodiazepine withdrawal program managed by primary care nurses in Spain
}

\author{
Cristina Lopez-Peig ${ }^{1,6}$, Xavier Mundet $^{2,3^{*}}$, Bartomeu Casabella ${ }^{1}$, Jose Luis del Val ${ }^{2}$, David Lacasta ${ }^{4}$ \\ and Eduard Diogene 3,5
}

\begin{abstract}
Background: Benzodiazepine (BZD), the long-term treatment of which is harmful for cognitive function, is widely prescribed by General Practitioners in Spain. Based on studies performed in other countries we designed a nurse-led BZD withdrawal program adapted to Spanish Primary Care working conditions.

Results: A pseudo-experimental (before-after) study took place in two Primary Care Centres in Barcelona. From a sample of 1150 patients, 79 were identified. They were over 44 years old and had been daily users of BZD for a period exceeding six months. Out of the target group 51 patients agreed to participate. BZD dosage was reduced every 2-4 weeks by $25 \%$ of the initial dose with the optional support of Hydroxyzine or Valerian. The rating measurements were: reduction of BZD prescription, demographic variables, the Short-Form Health Survey (SF-12) to measure quality of life, the Medical Outcomes Study (MOS) Sleep Scale, and the Goldberg Depression and Anxiety Scale.

By the end of the six-month intervention, 80.4\% of the patients had discontinued BZD and 64\% maintained abstinence at one year. An improvement in all parameters of the Goldberg scale $(p<0.05)$ and in the mental component of SF-12 at 3.3 points $(p=0.024)$, as well as in most components of the MOS scale, was observed in the group that had discontinued BZD. No significant differences in these scales before and after the intervention were observed in the group that had not discontinued.

Conclusions: At one year approximately $2 / 3$ of the patients had ceased taking BZD. They showed an overall improvement in depression and anxiety scales, and in the mental component of the quality of life scale. There was no apparent reduction in the sleep quality indicators in most of the analysed components. Nurses in a Primary Care setting can successfully implement a BZD withdrawal program.
\end{abstract}

Keywords: Benzodiazepine, Habituation drug therapy, Primary care

\section{Background}

Long-term treatment with Benzodiazepine (BZD) is harmful at varying cognitive levels [1]. Even though there is a clear association between the use of BZD by the elderly and an increased risk of falls, fractures, and road traffic accidents BZD is still widely prescribed by General Practitioners in Spain [2-4]. In fact, BZD is taken by up to $10 \%$ of the population in most developed countries [5].

Time constraints and a general lack of motivation in dealing with mental health issues on the part of General

\footnotetext{
*Correspondence: Xavier.Mundet@uab.cat

Unitat de Suport de la Recerca Barcelona Ciutat - IDIAP Jordi Gol, redIAPP Institut Català de la Salut, Sardenya 375, Barcelona 08025, Spain

${ }^{3}$ Universitat Autònoma de Barcelona, Bellaterra 08193, Spain

Full list of author information is available at the end of the article
}

Practitioners have contributed significantly to the elevated and inappropriate use of BZD in Primary Care [6]. Our study attempted to address this issue by giving nursing professionals (as opposed to General Practitioners) the leading role in managing BZD withdrawal programmes. Our final objective was to achieve a nurseled success rate for the programme equivalent to that obtained by General Practitioners.

Due to the fact that the number of studies carried out in Spain focusing on BZD withdrawal is very limited, our guidelines were based on strategies employed by General Practitioners in other countries. After reviewing the literature on BZD withdrawal programs, and using the study by Curran et al. in the United Kingdom as a main reference [7], we designed a programme to be managed by nursing professionals in Spanish Primary

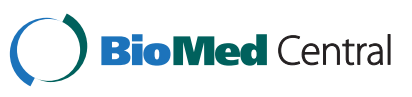


Care. Our aim was to demonstrate that (a) those patients who volunteered to participate would cease BZD consumption after six months, and that abstinence would be maintained for at least a further six months (twelve months in total), and (b) reduction of BZD was not harmful for the patient.

\section{Methods}

\section{Study design}

This is a before-after pseudo experimental study conducted in two large Primary Care Centres based in Barcelona (Spain). It was approved by the local Institut Català de la Salut-Barcelona ethics committee.

\section{Participants}

\section{Nurses}

Five qualified Primary Care nurses with varying levels of clinical experience agreed to participate. They were given training with respect to BZD usage and withdrawal programs in two 2-hour seminars which covered the following main topics: "Normal Sleep Physiology", "Sleep Hygiene and Insomnia", "Benzodiazepine History", and "Models and Therapies for Withdrawal Programs". The nurses were trained in assessing patients' needs for pharmacological support so they could choose, at their clinical discretion, to give either a prescription of Hydroxyzine ( $25 \mathrm{mg}$ per day) or advice on using over-thecounter Valerian products which have good tolerance, low risk, and reduced cost. Finally, they were provided with a thorough explanation of our protocol.

\section{Patients}

We identified patients of both sexes, over 44 years of age, who had used BZD daily for a period of more than 6 months. Participation was voluntary and written informed consent was obtained. Exclusion criteria were: epilepsy, cognitive impairment or dementia, hearing loss, severe visual defects, palliative care, and severe psychiatric illness. In addition, patients whose General Practitioner considered that their BZD withdrawal would be not be clinically appropriate were excluded.

\section{Protocol and baseline measures}

In a similar manner to Curran et al. we assessed several functions, including quality of life and sleep. We additionally employed The Goldberg Scale for Depression and Anxiety to better understand BZD usage. Patients who met the inclusion criteria were informed by their doctor about the study and the benefits of ceasing BZD. If they agreed to participate they were asked to complete a socio-demographic data collection questionnaire. Their responses were then evaluated by nurses who conducted a semi-structured interview assessing their attitudes and beliefs about BZD and sleep. In addition to gathering administrative data from the questionnaire, the nurses described the risks and benefits of long term BZD consumption, side effects, and possible dependence. They also briefly explained the programme and the implications of participating in the study. Three self-administered questionnaires were given to patients to be completed at home: The Short-Form Health Survey (SF-12) to measure quality of life $[8,9]$, the Medical Outcomes Study (MOS) sleep scale [10], and the Goldberg Depression and Anxiety scale [11]. Finally, a date was fixed to commence the withdrawal program. The general outline of the intervention program and each visit is summarized in Table 1.

Patients were evaluated by nurses every four weeks for the first 12 weeks. An assessment with the Goldberg Depression and Anxiety Scale, the quality of life scale (SF-12), and MOS sleep scale was performed at the initial visit, and at 12 and 24 weeks.

With regard to medication cessation, the baseline was the same in all cases. Patients could choose to continue taking either the same BZD or the equivalent dose of Diazepam which was then reduced by $25 \%$ every $2-4$ weeks. If, according to the nurse's assessment, pharmacological support with Hydroxyzine (25 $\mathrm{mg}$ per day) or Valerian was needed, the doctor would then be consulted. The doctor's supervisory role was to review the nurse's clinical decision, check the medication plan, and issue a prescription, if appropriate. The nurses also had the opportunity to provide feedback to the doctor. Information was extracted from patients' notes, surveys, and questionnaires. The primary endpoint (no consumption of BZD after six and twelve months) variable was obtained verbally from the patient and confirmed by prescription data.

\section{Study outcome measures}

The following variables were collected: socio demographic, toxic habits, antidepressant consumption, comorbidity, use of BZD, and data from the assessment scales: the Goldberg Depression and Anxiety Scale, The Short-Form Health Survey (SF-12), and The Medical Outcomes Study (MOS) sleep scale.

\section{Statistical analysis}

A data paired sample was calculated. Taking as a reference the results from various studies, a minimum BZD cessation of $20 \%$ was considered clinically relevant. Therefore, 40 patients (alpha error of 0.05 and beta error of 0.2 ) were required to give a statistically significant result. Mean with SDs and proportions were calculated for all variables. The Chi-square test was used to compare categorical data, and an unpaired Student t test was used to compare continuous variables. A two-tailed value of $\mathrm{P}<0.05$ was considered statistically significant. Cohen's $d$ 
Table 1 Intervention and data collection

\begin{tabular}{|c|c|}
\hline \multirow[t]{5}{*}{ Visit Selection } & Selection of candidates for study \\
\hline & Patient: Completes questionnaire. \\
\hline & Doctor: Checks that patient meets criteria for study (reviews results of data collection questionnaire + nurse interview). \\
\hline & Nurse: Semi-structured interview with patient. Consent obtained from patient. \\
\hline & If patient accepts: Questionnaires / test (T) given to patient + set up of start date (VISIT 0) \\
\hline Visit 0 & Collect questionnaires / tests + Brief educational intervention (BEI) + detoxification regimen given to patient \\
\hline Visit 4 & BEI + detoxification regimen plan given \\
\hline Visit 8 & BEI + detoxification regimen + Questionnaires / test $(\mathrm{T})$ given to patient \\
\hline Visit 12 & BEI + Collect questionnaires / tests and new Questionnaires / test (T) given to patient \\
\hline Visit 24 & $\mathrm{BEI}+$ Collect questionnaires / tests $(\mathrm{T})$ \\
\hline
\end{tabular}

BEl: Brief educational Intervention.

T: test. Include: SFHF 12, Goldberg, and MOS.

measured the effect size and compared it across the different variables.

\section{Results}

1150 patients were attended by their Primary Care doctors over an inclusion period of 3 months. From the 79 patients who met the inclusion criteria, 51 agreed to participate in the study (36 females and 15 males) (Figure 1). The mean age was 70.4 years (95\% CI: 66.4 to 73.9 years). Sociodemographic characteristics are presented in Table 2.
Regarding long term consumption, 22 patients (43.1\%) had been taking BZD for over 6 years, 21 patients (41.2\%) between 1 and 6 years, and 8 patients (15.7\%) for less than 1 year.

25 patients (50\%) began treatment with BZD due to insomnia, with an average consumption period of 5.12 years; 9 patients (18\%) were taking BZD due to anxiety, with an average consumption period of 10.67 years, and 5 patients (10\%) were depressed, with an average consumption period of 4.15 years. 11 patients $(22 \%)$ were

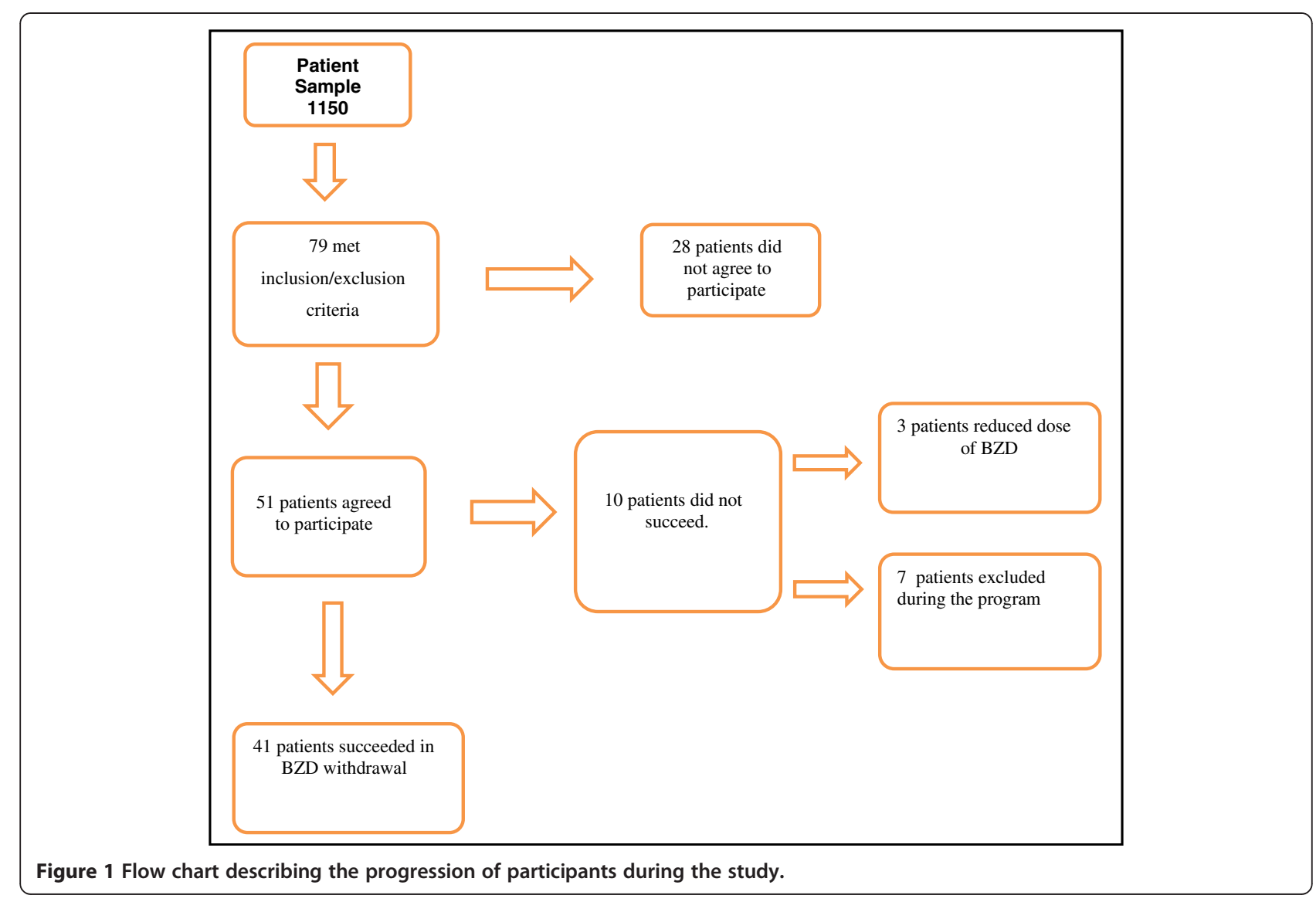


Table 2 Patients' features

\begin{tabular}{|c|c|}
\hline Features & $\mathrm{N}-(\%)$ \\
\hline Natives or residents for more than 5 years & $49(96.1)$ \\
\hline \multicolumn{2}{|l|}{ Education: } \\
\hline Primary school & $14(27.5)$ \\
\hline Secondary school & $34(66.7)$ \\
\hline University or postgraduate. & $3(5.9)$ \\
\hline \multicolumn{2}{|l|}{ Employment situation: } \\
\hline Pensioners & $36(70.6)$ \\
\hline Housewives & $6(11.8)$ \\
\hline Active workers & $9(17.6)$ \\
\hline \multicolumn{2}{|l|}{ Consumption of drugs: } \\
\hline Did not use antidepressants & $42(82)$ \\
\hline Consumed antidepressants: & $9(18)$ \\
\hline a) SSRI & $7(14)$ \\
\hline b) MAO & $1(2)$ \\
\hline c)Tricycle Antidepressants & $1(2)$ \\
\hline \multicolumn{2}{|l|}{ Chronic disease: } \\
\hline One or more chronic diseases & $43(84.3)$ \\
\hline COPD & $10(19.6)$ \\
\hline Osteoarthritis & $6(11.8)$ \\
\hline \multicolumn{2}{|l|}{ Toxic habits: } \\
\hline Alcohol & $2(3.9)$ \\
\hline Smoking & $13(25.5)$ \\
\hline Other drugs & $1(2)$ \\
\hline \multicolumn{2}{|l|}{ Cause of BZD consumption } \\
\hline Sleep disorder & $25(0.5)$ \\
\hline Anxiety & $9(0.2)$ \\
\hline Depression & $5(0.1)$ \\
\hline Other diagnoses & $11(0.2)$ \\
\hline
\end{tabular}

labelled as "other diagnoses" such us bereavement. divorce, and somatization.

46 patients remembered why BZD had been originally prescribed, with an average consumption period of 7.11 years. In contrast, 5 patients could not recall the reason for their original prescription, with an average consumption period of 4 years.

Among the patients who agreed to participate in the study 40 (78.4\%) were taking short-acting BZD, 8 (15.7\%) were using long acting BZD, and 3 (5.9\%) took other non-barbiturate hypnotics known as Z-drugs. The BZD most consumed in our study was $1 \mathrm{mg}$ of Lorazepam (58\%), equivalent to 10.4 of Diazepam (Figure 2).

The amount of information conveyed by the physician to the patient, when the prescription was first issued, was nil in the case of 31 patients, minimal for 13 patients, and only partial for the remaining 7 patients.
By the end of the intervention program, $80.4 \%$ of the patients had stopped their BZD intake and $64.7 \%$ maintained abstinence for one year.

In the analysis of the Short-Form Health Survey (SF-12) at baseline the mental component (45.96) consistently achieved a higher score than the physical one (39.60). Both results showed scores below the population mean (50).

Within the group of patients who ceased BZD, comparative analysis of the SF-12 before the intervention and at 24 weeks showed an improvement of 3.3 points $(\mathrm{p}=0.02)$ in the mental component (Table 3$)$. The same comparative analysis of its physical component was made, but no statistically significant differences were observed. Within the group of non-compliance there were no statistically significant improvements in any of the physical or mental components.

From analysis of the scales (Table 3) it can be seen that in the MOS sleep scale, those patients taking BZD chronically obtained, at the beginning, a higher score in all parameters of sleep analysis than the population mean. The average hours of sleep was 6.10 (SD 1.46), and 16 patients slept an adequate number of hours (7-8 hours). Within the compliant group results from the same scale showed no difference in most of the components throughout the intervention. Two exceptions were deterioration in snoring perception and an increase in breathing difficulty. Within the non-compliance group there were no statistically significant differences.

Analysis of the Goldberg Depression and Anxiety Scale at the beginning of the program showed an average score compatible with depression but not anxiety (breakpoints are $\geq 4$ for the anxiety scale and $\geq 2$ for depression). The unsuccessful group showed a lower mean in the depression component at baseline, but without statistically significant differences between the beginning and the end of the study. The same group also showed a higher mean in the anxiety score although still below the anxiety threshold.

\section{Discussion}

The study was designed to show that a BZD withdrawal program, with nursing professionals as the key element, can be applicable within the Spanish Primary Care setting. The following points were considered in developing our program: a) In agreement with many authors, patients should not be forced to cease their treatment [12]; b) The use of complementary medicines as coadjuvant therapy. Numerous trials have been carried out using complementary medicines to control withdrawal symptoms while the patients are on BZD [13-15]. These trials have highlighted the successful use of Hydroxyzine [16] due to its good tolerance, low risk, and reduced cost; c) Tapered dose. Other studies have also focused 


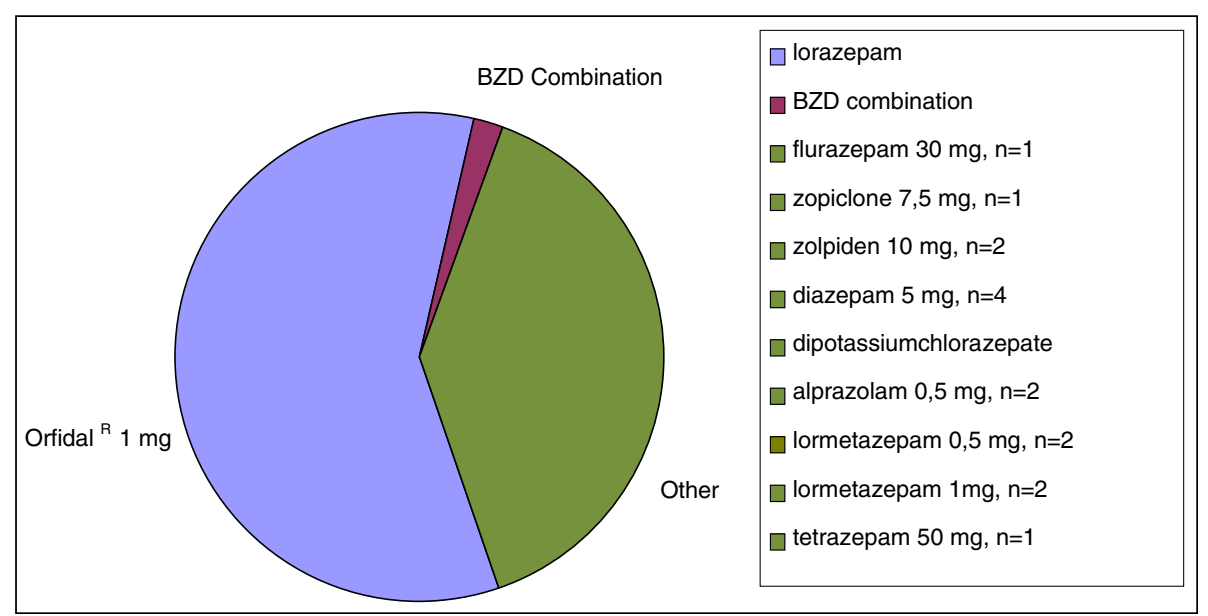

Figure 2 Types of BZD consumed by patients.

on a progressive cessation of BZD $[17,18]$, which reveal a cost-effective alternative for BZD withdrawal; d) Pharmacological support. Although cognitive behavioural therapy is an effective adjuvant therapy in BZD withdrawal programs $[19,20]$ its implementation in daily practice can have significant cost and time implications. Several clinical trials have, therefore, focused instead on providing psychological support to patients during their withdrawal period, based on identifying the reason prescriptions were initiated and maintained, and patients' information on dependence and withdrawal symptoms $[21,22]$; and e) The use of Valerian for improving sleep. Whilst Valerian has a subjective improvement on sleep quality it does not reflect an improvement in sleep onset latency [23].

After the level of commitment required to successfully complete the first stage of this programme (i.e. to attend at least six appointments with the nurse and to complete multiple assessment questionnaires) had been explained, 28 of the selected patients did not wish to participate. Most of these patients gave as their reason for refusal the fact of being in active employment and, therefore, limited in terms of time. It should also be noted that the study excluded young patients, which additionally reduced our patient sample. As a result, our sample group consisted predominantly of elderly and retired patients (particularly female pensioners with a low socio-economic level) with concomitant chronic diseases.

Their socio-demographic features matched those of patients defined as being at risk of chronic BZD use $[24,25]$.

Our sample size was large enough to show the beneficial effect of a BZD cessation programme and highlight the difference between the patients' condition before and after the intervention programme. We recognize, however, that size was the main limitation of the study, and we were unable to obtain statistically significant differences between patients who successfully completed the cessation programme and those who did not. The latter comparison was not, however, a key objective although we consider it could become a useful starting point for further studies.

It should be pointed out that almost $20 \%$ of the patients consumed antidepressants, mostly from the Selective Serotonin Reuptake Inhibitor (SSRI) group. This highlights non-compliance with national guidelines based on previous studies [26] which advise limiting the use of BZD to the onset of SSRI treatment. More than twenty years ago it was reported that the benefit of BZD was significantly reduced after fifteen days of use [27]. Yet, as our results confirm, BZD continues to be prescribed without due consideration for this benefit/time restriction.

A comparison revealed that the average daily dose of BZD in the United Kingdom (equivalent to $5 \mathrm{mg}$ of Diazepam) [7] was lower than that in our study in Spain (equivalent to $10 \mathrm{mg}$ of Diazepam). Nevertheless, the percentage of cessation at six months was the same in both countries. Results achieved in withdrawal programmes in both countries were not, therefore, influenced by the initial BZD dosage. In our study, patients taking a higher initial dose of BZD presented more sleeping problems in the baseline test than those taking lower doses; once, however, they had all completed the program, those who had initially consumed more BZD did not score higher on the sleeping problem scale. This finding helped to confirm that the consumption of a higher dose of BZD does not improve sleep quality $[7,28]$. It is noteworthy, that $10 \%$ of the patients who were taking BZD (with its concomitant side effects) $[2,29]$ in excess of four years were unaware of the reason for its indication. 
Table 3 Comparison of quality of life (SF-12), Goldberg, and MOS scales

\begin{tabular}{|c|c|c|c|c|c|c|c|}
\hline \multirow[b]{2}{*}{ Scale } & \multicolumn{3}{|c|}{ No success (12 pacs) } & \multicolumn{3}{|c|}{ Success (39 pacs) } & \multirow[b]{2}{*}{ Effect size Cohen's } \\
\hline & Mean & Desv. Est. & $p$ & Mean & Desv. Est. & $p$ & \\
\hline \multicolumn{8}{|c|}{ SF.12: physical component } \\
\hline 0 & 41.89 & 11.62 & & 39.60 & 8.82 & & 0.25 \\
\hline 12 months & 49.09 & 1.63 & 0.36 & 39.89 & 8.36 & 0.79 & 1.27 \\
\hline 24 months & 49.71 & 2.98 & 0.58 & 38.99 & 7.49 & 0.52 & 1.62 \\
\hline \multicolumn{8}{|c|}{ SF12: mental component } \\
\hline 0 & 42.79 & 16.12 & & 45.96 & 9.88 & & -0.28 \\
\hline 12 months & 48.03 & 11.17 & 0.28 & 48.79 & 9.74 & 0.08 & -0.08 \\
\hline 24 months & 40.72 & 19.63 & 0.43 & 49.27 & 9.73 & 0.02 & -0.69 \\
\hline \multicolumn{8}{|l|}{ Goldberg anxiety } \\
\hline 0 & 3.75 & 3.19 & & 3.23 & 2.65 & & 0.19 \\
\hline 12 months & 0.75 & 1,50 & 0,30 & 2,33 & 2,31 & 0,01 & -0.75 \\
\hline 24 months & 2,67 & 2,52 & 1,00 & 2,26 & 2,42 & 0,01 & 0.17 \\
\hline \multicolumn{8}{|c|}{ Goldberg depression } \\
\hline 0 & 2,17 & 1,85 & & 2,36 & 2,31 & & -0.09 \\
\hline 12 months & 1,00 & 1,41 & 0,42 & 1,62 & 2,42 & 0,00 & -0.28 \\
\hline 24 months & 1.00 & 1,73 & 0,42 & 1,55 & 1,84 & 0,00 & -0.31 \\
\hline \multicolumn{8}{|c|}{ Short of Breath Scale } \\
\hline 0 & 75,00 & 28,44 & & 82,56 & 24,36 & & -0.30 \\
\hline 12 months & 95,00 & 10,00 & 0,42 & 85,64 & 20,49 & 0,32 & 0.51 \\
\hline 24 months & 100,00 & 0,00 & 0,07 & 90,26 & 16,46 & 0,02 & 0.69 \\
\hline \multicolumn{8}{|l|}{ Sleep Adequacy } \\
\hline 0 & 40,00 & 24,12 & & 56,15 & 27,30 & & -0.62 \\
\hline 12 months & 30,00 & 34,64 & 0,07 & 56,41 & 22,18 & 0,96 & -1.06 \\
\hline 24 months & 53,33 & 11,55 & 0,06 & 58,46 & 26,31 & 0,36 & -0.22 \\
\hline \multicolumn{8}{|c|}{ Sleep Disturbance Scale } \\
\hline 0 & 51,88 & 14,19 & & 55,71 & 14,78 & & -0.27 \\
\hline 12 months & 68,44 & 10,87 & 0,19 & 56,60 & 13,02 & 0,73 & 0.96 \\
\hline 24 months & 57,92 & 6,88 & 0,68 & 57,31 & 12,15 & 0,44 & 0.06 \\
\hline \multicolumn{8}{|c|}{ Sleep Problems Index I } \\
\hline 0 & 61,39 & 14,32 & & 62,99 & 9,88 & & -0.15 \\
\hline 12 months & 75,00 & 15,99 & 0,46 & 63,85 & 6,86 & 0,60 & 1.17 \\
\hline 24 months & 67,78 & 6,94 & 0,15 & 65,47 & 6,69 & 0,07 & 0.35 \\
\hline \multicolumn{8}{|c|}{ Sleep Problems Index II } \\
\hline 0 & 59,35 & 11,42 & & 60,94 & 9,34 & & -0.16 \\
\hline 12 months & 71,53 & 12,86 & 0,40 & 61,45 & 5,54 & 0,83 & 1.32 \\
\hline 24 months & 62,04 & 6,99 & 0,47 & 62,68 & 6,61 & 0,18 & -0.10 \\
\hline \multicolumn{8}{|l|}{ Snoring Scale } \\
\hline 0 & 71,67 & 35,63 & & 66,15 & 37,32 & & 0.15 \\
\hline 12 months & 100,00 & 0,00 & 0,18 & 70,77 & 35,79 & 0,24 & 0.95 \\
\hline 24 months & 100,00 & 0,00 & 0,18 & 74,87 & 29,99 & 0,03 & 0.97 \\
\hline \multicolumn{8}{|l|}{ Somnolence Scale } \\
\hline 0 & 59,44 & 17,16 & & 73,50 & 17,54 & & -0.82 \\
\hline 12 months & 70,00 & 20,73 & 0,21 & 72,82 & 15,09 & 0,86 & -0.17 \\
\hline 24 months & 64,44 & 20,37 & 0,12 & 76,92 & 14,25 & 0,25 & -0.80 \\
\hline
\end{tabular}


When analysing the SF-12 results before the initial intervention and at 24 weeks, we saw an improvement in the mental component but not in the physical one among the cessation group. These results are similar to those expected as consumption of BZD should not influence physical fitness.

In the analysis of the Goldberg scale, the cessation group showed a statistically significant gradual decrease in both the anxiety and depression components. These results are positive when compared with the study by Curran et al. [7] which, whilst using a different parametric scale (the Geriatric Depression Scale), reported neither an improvement nor a deterioration of these symptoms for the cessation group.

The results of the sleep scale could be interpreted as being due to a heightened awareness of other underlying chronic diseases. In fact, a quarter of the patients had Chronic Obstructive Pulmonary Disease (COPD) and they should not have been taking BZD as a chronic treatment. Discontinuing BZD prevents severe symptoms being masked, and thus permits early action on potential complications. A $64.7 \%$ success rate (percentage of BZD cessation cases maintained for one year) shows that, after specific training, nurses in a Primary Care setting can implement a program with the objective of achieving the cessation of BZD intake in volunteer patients, with similar results to studies conducted by other professionals such as physicians or psychologists $[7,30,31]$. Nevertheless, it must be conceded that our outcome could be partly attributed to an inaccurate diagnosis of insomnia, mistaking this, and the patient's addiction due to tolerance [32], for physiological sleep disturbances related to age, which are easy to treat through advice and sleep hygiene. Our results may provide another overview [33] of the initial and medium term results of this withdrawal program and represent a starting point for further studies on effectiveness.

\section{Conclusions}

Our withdrawal program, conducted by nurses, was successful in that a period of one year. $2 / 3$ of the patients in our sample ceased taking BZD. These results are similar to studies conducted by other professionals such as physicians or psychologists. Our work confirms the fact that nurses in a Primary Care setting can successfully implement a BZD withdrawal program.

\section{Abbreviations}

BZD: Benzodiazepine; SF-12: Short-Form Health Survey; MOS: Medical Outcomes Study; SSRI: Selective Serotonin Reuptake Inhibitor; COPD: Chronic Obstructive Pulmonary Disease; MAOI: Mono Amin Oxidase Inhibitors.

\section{Competing interests}

The authors declare that they have no competing interest.

\section{Authors' contributions}

$\mathrm{CL}$ : Principal investigator, protocol design, seminar speaker, interpretation of the data, writing of the manuscript. XM: Study management, protocol design, data interpretation, writing of the manuscript. BC: Study management, protocol design, patient recruitment. JLV: Statistical analyses, data interpretation, writing of the manuscript. DL: Patient recruitment. ED: Study management, protocol design, data interpretation. All the authors have read and approved the final manuscript.

\section{Acknowledgements}

We wish to thank the nursing team working at Raval Sud and Maragall Primary Care Centers for their contributions to patient recruitment and data collection. Special thanks to Lola Garcia. We are also extremely grateful to the team at Woodberry Practice and Dr. Olatz Heredia for all their time and support.

\section{Author details}

${ }^{1}$ Primary Care Center Raval Sud. Institut Català de la Salut, Barcelona 08001, Spain. ${ }^{2}$ Unitat de Suport de la Recerca Barcelona Ciutat - IDIAP Jordi Gol, redIAPP Institut Català de la Salut, Sardenya 375, Barcelona 08025, Spain. ${ }^{3}$ Universitat Autònoma de Barcelona, Bellaterra 08193, Spain. ${ }^{4}$ Primary Care Center La Salut. Institut Català de la Salut, Badalona 08914, Spain. ${ }^{5}$ Institut Català de Farmacologia, Barcelona, Spain. ${ }^{6}$ Woodberry Practice. Winchmore Hill. Enfield. NHS trust, London, UK.

Received: 19 March 2012 Accepted: 26 November 2012

Published: 13 December 2012

\section{References}

1. Rummans TA, Davis $L \mathrm{Jr}$, Morse RM, IvniK RJ: Learning and memory impairment in older, detoxified, benzodiazepine-dependent patients. Mayo Clin Proc 1993, 68:731-737.

2. Thomas RE: Benzodiazepine use and motor vehicle accidents. Systematic review of reported associaton. Can Fam Physician 1998, 44:799-808.

3. Leipzig RM, Cumming RG, Tinetti ME: Drugs and fall in older people: a systematic review and meta-analysis: I Psychotropic drugs. J Am Geriatr Soc 1999, 47:30-39.

4. Madhusoodanan S, Bogunovic OJ: Safety of benzodiazepines in the geriatric population. Expert Opin Drug Saf 2004, 3:485-493.

5. Rang HP, Dale MM, Ritter JM: Pharmacology. 4ath edition. London: Harcourt Publishers Limited; 2000.

6. Cormack MA, Howells E: Factors linked to the prescribing of benzodiazepines by general practice principals and trainees. Fam Pract 1992, 9:466-471.

7. Curran HV, Collins R, Fletcher S, Kee SC, Woods B, Lliffe S: Older adults and withdrawal from benzodiazepine hypnotics in general practice: effects on cognitive function, sleep, mood and quality of life. Psychol Med 2003, 33:1223-1237.

8. Alonso J, Prieto L, Antó JM: La versión española del SF-36 Health Survey (Cuestionario de Salud SF-36): un instrumento para la medida de los resultados clínicos. Med Clin (Barc) 1995, 104:771-776.

9. Vilagut G, Ferrer M, Rajmil M, Rebollo P, Permanyer-Miralda G, Quintana JM, Santed R, Valderas JM, Ribera A, Domingo-Salvany A, Alonso J: El cuestionario de salud SF-36 español: una década de experiencia y nuevos desarrollos. Gac Sanit 2005, 19(2):135-150.

10. Hays RD, Stewart AL: Sleep measures. In Measuring functioning and well-being: The Medical Outcomes Study approach. Edited by Stewart AL, Ware JE. Durham, NC: Duke University Press; 1992:235-259.

11. Montón C, Pérez-Echeverria M, Campos R, cols y: Escalas de ansiedad y depresión de Goldberg: una guía de entrevista eficaz para la detección del malestar psíquico. Aten Primaria 1993, 12(6):345-349.

12. Ashton H: The treatment of benzodiazepine dependence. Addiction 1994, 89:1535-1541.

13. Linden M, Bar T, Geiselmann B: Patient treatment insistence and medication craving in long-term low-dosage benzodiazepine prescriptions. Psychol Med 1998, 28:721-729.

14. Croissant B, Grosshans M, Klein O, Diehl A, Mann K: Scheme-based benzodiazepine detoxification with oxcarbazepine a case report. Pharmacopsychiatry 2005, 38:222-223. 
15. Finkel JC, Johnson YJ, Quezado ZM: The use of dexmedetomidine to facilitate acute discontinuation of opioids after cardiac transplantation in children. Crit Care Med 2005, 33:2110-2112.

16. Lemoine P, Touchon J, Billardon M: Comparison of 6 different methods for lorazepam withdrawal. A controlled study, hydroxyzine versus placebo. Encéphale 1997, 23:290-299.

17. Rosenbaum JF: Attitudes toward benzodiazepines over the years. J Clin Psychiatry 2005, 66(2):48.

18. McGregor C, Machin A, White JM: In-patient benzodiazepine withdrawal: comparison of fixed and symptom-triggered taper methods. Drug Alcohol 2003, 22:175-180.

19. Morin CM, Bastien C, Guay B, Radouco-Thomas M, Leblanc J, Vallieres A: Randomized clinical trial of supervised tapering and cognitive behavior therapy to facilitate benzodiazepine discontinuation in older adults with chronic insomnia. Am J Psychiatry 2004, 161:332-342.

20. Baillargeon L, Landreville P, Verreault R, Beauchemin JP, Gregoire JP, Morin CM: Discontinuation of benzodiazepines among older insomniac adults treated with cognitive-behavioural therapy combined with gradual tapering: a randomized trial. CMAJ 2003, 169:1015-1020

21. Landry P, Mainguy N: Clinical advice facilitating withdrawal of benzodiazepines. Sante Ment Que 2003, 28:43-58.

22. Khong E, Sim MG, Hulse G: Benzodiazepine dependence. Aust Fam Physician 2004, 33:923-926.

23. Poyares DR, Guilleminault C, Ohayon MM, Tufik S: Can valerian improve the sleep of insomniacs after benzodiazepine withdrawal? Prog Neuropsychopharmacol Biol Psychiatry 2002, 26:539-545.

24. O'Connor K, Belanger L, Marchand A, Dupuis G, Elie R, Boyer R: Psychological distress and adaptational problems associated with discontinuation of benzodiazepines. Addict Behav 1999, 24:537-541.

25. O'Connor KP, Marchand A, Belanger L, Mainguy N, Landry P, Savard P, Turcotte J, Dupuis G, Harel F, Lachance L: Psychological distress and adaptational problems associated with benzodiazepine withdrawal and outcome: a replication. Addict Behav 2004, 29:583-593.

26. Fourrier A, Letenneur L, Dartiajes JF, Moore N, Begaud B: Benzodiazepine use in an elderly community-dwelling population. Characteristics of users and factors associated with subsequent use. Eur J Clin Pharmacol 2001, 57:419-425.

27. Lader $\mathrm{M}$, Tylee $\mathrm{A}$, Donoghue J: Withdrawing benzodiazepines in primary care. CNS Drugs 2009, 23(1):19-34.

28. De Las C, Sanz E, De La Fuente J: Benzodiazepines: More behavioral addiction than dependence. Psychopharmacology 2003, 167:297-303.

29. Leipzig RM, Cumming RG, Tinetti ME: Drugs and fall in older people: a systematic review and meta-analysis: I. Psychotropic drugs. Journal of American Geriatric. Society 1999, 47:30-39.

30. Oude Vosahar RC, Couvee JE, Van Balkom A, Mulder P, Zitman FG: Strategies for discontinuing long term benzodiazepine use. Br J Psych 2006, 189:213-220.

31. Taylor DJ, Schmidt-Nowara W, Jessop CA, Ahearn J: Sleep restriction therapy and hypnotic withdrawal versus sleep hygiene education in hypnotic using patients with insomnia. J Clin Sleep Med 2010, 6(2):169-175.

32. Higgitt $\mathrm{A}$, Fonagy $\mathrm{P}$, Lader $\mathrm{M}$ : The natural history of tolerance to the benzodiazepines. Psychol Med Monogr Suppl 1988, 13:1-55.

33. Vicens C, Fiol F: Abordaje de la deshabituación de benzodiacepinas en atención primaria. Inf Ter Sist Nac Salud 2008, 32:52-57.

\section{doi:10.1186/1756-0500-5-684}

Cite this article as: Lopez-Peig et al:: Analysis of benzodiazepine withdrawal program managed by primary care nurses in Spain. BMC Research Notes 2012 5:684.

\section{Submit your next manuscript to BioMed Central and take full advantage of:}

- Convenient online submission

- Thorough peer review

- No space constraints or color figure charges

- Immediate publication on acceptance

- Inclusion in PubMed, CAS, Scopus and Google Scholar

- Research which is freely available for redistribution

Submit your manuscript at www.biomedcentral.com/submit
Ciomed Central 